\title{
Efecto del $1 \alpha$-hidroxicolecalciferol sobre el comportamiento productivo de pollos de engorde en condiciones de crianza comercial
}

\author{
Effect of $1 \alpha$-hydroxycholecalciferol on the productive performance of broilers under commercial \\ conditions
}

\author{
Gabriela Peceros Ripa ${ }^{1}$; Florencio Eusebio Capcha ${ }^{2}$; Carlos Vilchez Perales ${ }^{3}$
}

Resumen

El objetivo del presente estudio fue evaluar la suplementación de la vitamina $1 \alpha$-hidroxicolecalciferol $\left(1 \alpha-\mathrm{OHD}_{3}\right)$ en dietas para pollos de carne a través de su comportamiento productivo e incidencia de problema de patas. Para ello, se usaron 16.290 pollos machos de la línea Cobb 500 de un día de edad, distribuidos al azar en tres grupos de 5.430 aves cada uno; cada grupo fue alimentado ad libitum durante 41 días. Los tratamientos fueron: T1, Dieta control positivo; T2, Dieta deficiente en calcio y fósforo $+125 \mathrm{~g}$ de $1 \alpha-\mathrm{OHD}_{3}$ por tonelada métrica y T3, Dieta control negativo (Similar a T2, pero sin la adición de $1 \alpha-\mathrm{OHD}_{3}$ ). Los resultados mostraron que el comportamiento productivo de los pollos de carne alimentados con dietas conteniendo niveles inferiores de calcio y fósforo a los requeridos, pero suplementados con vitamina $1 \alpha-\mathrm{OHD}_{3}(\mathrm{~T} 2)$ fue similar al del grupo control positivo (T1), mientras que la retribución económica fue favorable en el grupo que fue alimentado con la dieta suplementada con $1 \alpha-\mathrm{OHD}_{3}$ (T2) en comparación a con los otros dos tratamientos. En conclusión, los pollos de carne alimentados con dietas deficientes en calcio y fósforo, pero suplementados con $1 \alpha-\mathrm{OHD}_{3}$ presentaron similares respuestas productivas que los del grupo de pollos que fueron alimentados con la dieta control positivo.

Palabras clave: $1 \alpha$-hidroxicolecalciferol; comportamiento productivo; pollos de carne; retribución económica.

\begin{abstract}
The objective of the present study was to evaluate the effect of vitamin $1 \alpha$-hidroxicolecalciferol $\left(1 \alpha-\mathrm{OHD}_{3}\right)$ in broiler diets as measured by performance and incidence of leg problems. On day of hatch, 16.290 male Cobb 500 broiler chicks were randomly and equally allocated to three groups of 5.430 birds each and were fed ad libitum for 41 days. Treatments were: T1, Positive Control Diet; T2, Deficient diet in calcium and phosphorus $+125 \mathrm{~g}$ of $1 \alpha-\mathrm{OHD}_{3}$ per metric ton; T3, Negative Control Diet (similar to T2 but without the addition of $1 \alpha$-hidroxicolecalciferol). Results showed that the productive performance of chickens fed with deficient dietary levels of calcium and phosphorus, but supplemented with vitamin $1 \alpha-\mathrm{OHD}_{3}(\mathrm{~T} 2)$ was similar to the results of the positive control group (T1), while the economic retribution was favorable for the group fed with the supplemented $1 \alpha-\mathrm{OHD}_{3}(\mathrm{~T} 2)$ diet, compared with the other two treatments. In conclusion, broilers fed diets deficient in calcium and phosphorus but supplemented with $1 \alpha-\mathrm{OHD} 3$ showed similar productive responses than those in the group of broilers fed with the positive control diet.
\end{abstract}

Keywords: $1 \alpha$ - hydroxycholecalciferol; productive performance; broilers; economic retribution.

\section{Introducción}

En la actualidad, la alimentación de las aves en el Perú está basada mayormente en la utilización del maíz y de la torta de soya debido a que son buenas fuentes energéticas y proteicas; sin embargo, estos ingredientes contienen bajas concentraciones de fósforo y calcio. La mayor parte del fósforo de estos ingredientes se encuentra en forma de ácido fítico, un compuesto que resulta poco digerible para las aves. Como el calcio y el fósforo son importantes para el organismo de las aves, los avicultores se ven en la necesidad de añadir estos minerales como suplementos inorgánicos en los alimentos balanceados. Después de los ingredientes energéticos y proteicos, son los ingredientes inorgánicos, particularmente los fosfatos, los más costosos; por tanto el uso de aditivos nutricionales o no nutricionales que mejoren la utilización del calcio y del fósforo de los ingredientes de la dieta, como la vitamina $1 \alpha$-hidroxicolecalciferol $\left(1 \alpha-\mathrm{OHD}_{3}\right)$, podría permitir la disminución del uso de suplementos inorgánicos y reducirían, de esta manera, el costo de la dieta y de redueir la excreción de fósforo en el medio ambiente.

Los primeros en evaluar la $1 \alpha-\mathrm{OHD}_{3}$ en dietas para pollos

\footnotetext{
Ingeniero Zootecnista. Práctica privada

$\mathrm{Mg}$. Sc. Consultor Nutricional. Práctica privada.

Profesor Principal, Departamento Académico de Nutrición, Facultad de Zootecnia, Universidad Nacional Agraria La Molina. Lima, Perú. E-mail: cvilchezp@lamolina.edu.pe
} 
de carne fueron Haussler et al. (1973), quienes confirmaron que la actividad biológica de la $1 \alpha-\mathrm{OHD}_{3}$ es similar a la 1,25-dihidroxicolecalciferol $\left(1,25-(\mathrm{OH})_{2} \mathrm{D}_{3}\right) \mathrm{y}$, además, sugirieron que la $1 \alpha-\mathrm{OHD}_{3}$ luego de ser absorbida en el intestino, es hidroxilada rápidamente en el carbono-25, con el fin de convertirse en $1,25-(\mathrm{OH})_{2} \mathrm{D}_{3}$. (a parte)

La 1,25-(OH) $\mathrm{D}_{3}$ es la forma biológicamente más activa de la vitamina $\mathrm{D}_{3}$ y se relaciona con la absorción de calcio y fósforo, así como con el mantenimiento de la homeostasis de estos minerales en el organismo de las aves (Leeson y Summers, 2001). Varias investigaciones confirmaron que la $1 \alpha-\mathrm{OHD}_{3}$ actúa como precursor de la $1,25-(\mathrm{OH})_{2} \mathrm{D}_{3}$ (Biehl et al., 1998; Edwards, Jr., 2002; Han et al., 2009). En pollos de carne, la $1 \alpha-\mathrm{OHD}_{3}$ es ocho veces más potente en reducir la incidencia de raquitismo, incrementar los pesos corporales y cenizas de hueso que la vitamina $\mathrm{D}_{3}$ (Edwards et asl., 2002). La potencia de la $1,25(\mathrm{OH})_{2} \mathrm{D}_{3}$ es levemente mayor o similar que la $1 \alpha-\mathrm{OHD}_{3}$ para reducir la incidencia de raquitismo, aumentar cenizas del hueso, retención de calcio, fósforo y fósforo-fítico (Edwards, 2002).

La eficacia y requerimiento de la $1 \alpha-\mathrm{OHD}_{3}$ varía de acuerdo at con el parámetro evaluado y al nivel de calcio y/o fósforo en la dieta. Biehl y Baker (1997) reportaron incrementos de pesos corporales y cenizas de hueso cuando la $1 \alpha-\mathrm{OHD}_{3}$ se usa como suplemento en dietas a base de maíz-soya y deficientes en calcio y fósforo, pero estas respuestas disminuyeron cuando se incrementó el nivel de calcio en la dieta. Cuando los niveles de calcio en la dieta son altos

Tabla 1. Composición porcentual de las dietas experimentales de pre-inicio (1-7 días) e inicio (8-21 días)

\begin{tabular}{|c|c|c|c|c|c|c|}
\hline \multirow{2}{*}{ Ingredientes } & \multicolumn{3}{|c|}{ Pre-inicio (1 - 7 días) } & \multicolumn{3}{|c|}{ Inicio (8 - 21 días) } \\
\hline & $\mathbf{T}^{1}{ }^{1}$ & $\mathbf{T} 2$ & T3 & T1 & $\mathbf{T 2}$ & T3 \\
\hline Maíz amarillo & 59,51 & 59,41 & 59,41 & 61,56 & 6,83 & 61,83 \\
\hline Torta soya (paraguaya) & 28,69 & 28,61 & 28,61 & 30,82 & 31,13 & 31,13 \\
\hline Harina de soya integral & - & - & - & 3,576 & 3,113 & 3,126 \\
\hline Gluten maíz & 5 & 5 & 5 & - & - & - \\
\hline Aceite de maíz & - & - & - & 0,5 & 0,5 & 0,5 \\
\hline Subproducto de trigo & 2,45 & 2,767 & 2,78 & - & - & - \\
\hline Fosfato tricálcico & 1,34 & 1,06 & 1,06 & 1,23 & 0,96 & 0,96 \\
\hline Carbonato de calcio & 1,06 & 1,16 & 1,16 & 0,66 & 0,77 & 0,77 \\
\hline Sal común & 0,25 & 0,27 & 0,27 & 0,18 & 0,21 & 0,21 \\
\hline Premezcla vitamínico mineral & 1,25 & 1,25 & 1,25 & 1,25 & 1,25 & 1,25 \\
\hline Lisina HCL & 0,17 & 0,17 & 0,17 & - & - & - \\
\hline L- Treonina & 0,05 & 0,05 & 0,05 & 0,004 & 0,004 & 0,004 \\
\hline Cloruro de colina 75 & 0,13 & 0,13 & 0,13 & 0,11 & 0,11 & 0,11 \\
\hline Enzima liquido (Vegpro) & 0,05 & 0,05 & 0,05 & 0,05 & 0,05 & 0,05 \\
\hline Acido propiónico 50 & 0,05 & 0,05 & 0,05 & - & - & - \\
\hline Bicarbonato sodio & - & 0,01 & 0,01 & - & - & - \\
\hline Vitamina 1alpha-OHD 3 & - & 0,0125 & - & - & 0,0125 & - \\
\hline \multicolumn{7}{|l|}{ Nutrientes (Calculado) } \\
\hline E. Metabolizable (kcal/kg) & 2.940 & 2.940 & 2.940 & 3.040 & 3.040 & 3.040 \\
\hline Proteína total, \% & 22 & 22 & 22 & 21,5 & 21,5 & 21,5 \\
\hline Lisina total, \% & 1,38 & 1,38 & 1,38 & 1,33 & 1,33 & 1,33 \\
\hline Metionina total, $\%$ & 0,63 & 0,63 & 0,63 & 0,56 & 0,56 & 0,56 \\
\hline Metionina + Cistina total, $\%$ & 1,00 & 1,00 & 1,00 & 0,91 & 0,91 & 0,91 \\
\hline Triptófano total, $\%$ & 0,24 & 0,24 & 0,24 & 0,27 & 0,27 & 0,27 \\
\hline Treonina total, \% & 0,97 & 0,97 & 0,97 & 0,94 & 0,94 & 0,94 \\
\hline Calcio, $\%$ & 1,00 & 0,95 & 0,95 & 0,9 & 0,85 & 0,85 \\
\hline Fósforo disponible, \% & 0,48 & 0,43 & 0,43 & 0,46 & 0,41 & 0,41 \\
\hline Sodio, $\%$ &, 22 & 0,22 & 0,22 & 0,18 & 0,18 & 0,18 \\
\hline
\end{tabular}

1 Tratamientos: T1: control positivo; T2: dieta conteniendo $1 \alpha-\mathrm{OHD}_{3} ; \mathbf{T 3}$ : control negativo. 
es posible no observar beneficios de la $1 \alpha-\mathrm{OHD}_{3}$ sobre los pesos corporales, este efecto posiblemente se debe- deba a por una condición de hipercalcemia que se presenta en los pollos (Liem et al., 2009).

Asimismo, Han et al., (2009) indican que el efecto que produce la $1 \alpha-\mathrm{OHD}_{3}$ sobre los pollos de carne varía según el nivel de fósforo no fítico (fósforo disponible) en la dieta. Así, la suplementación de $5 \mu \mathrm{g}$ de $1 \alpha-\mathrm{OHD}_{3} / \mathrm{kg}$ de alimento en dietas deficientes en fósforo total $(0,44 \%$ $\mathrm{P}$ total), fósforo disponible $(0,21 \% \mathrm{P}$ disponible) y con niveles adecuados de calcio pueden incrementar pesos corporales, eficiencia alimenticia, resistencia a la rotura de huesos, utilización del fósforo total y confirmaron que la $1 \alpha-\mathrm{OHD}_{3}$ incrementa la transmisión de genes para sintetizar proteínas transportadoras de fosfato (NaPi-IIb) en el intestino delgado.

En otro estudio, en dietas conteniendo un mayor nivel de fósforo disponible $(0,30 \% \mathrm{P}$ disponible $)$, con $5 \mu \mathrm{g}$ de $1 \alpha-\mathrm{OHD}_{3} / \mathrm{kg}$ de alimento y bajos niveles fósforo total $(0,50$ $\% \mathrm{P}$ total) y calcio $(0,72 \% \mathrm{Ca})$ no se reportó incremento sobre los pesos corporales, pero hubo una disminución en la incidencia de raquitismo, incremento sobre la cantidad de cenizas de hueso y retención de calcio y fósforo (Edwards, Jr., 2002). Por otro lado, la suplementación de $1 \alpha-\mathrm{OHD}_{3}$ en dietas a base de maíz-soya y conteniendo adecuada cantidad de vitamina $\mathrm{D}_{3}$ incrementa la utilización del fósforo fítico en pollos de carne (Biehl et al., 1998; Edwards, Jr., 2002; Snow et al., 2004; Shim et al., 2008). A pesar de estos resultados, el mecanismo por el cual la $1 \alpha-\mathrm{OHD}_{3}$ incrementa la utilización del acido fítico es incierto (Biehl et al., 1998; Han et al., 2009).

La mayoría de las investigaciones reportadas se llevaron a cabo en condiciones de laboratorio, con dietas maízsoya y con un limitado número de aves. Por lo tanto, el presente estudio tiene como objetivo determinar el efecto de la suplementación de $1 \alpha-\mathrm{OHD}_{3}$ en dietas complejas sobre el comportamiento productivo, problemas de patas y mortalidad de pollos de carne criados en condiciones comerciales.

\section{Materiales y métodos}

El presente estudio se llevó a cabo en las instalaciones de la empresa Gramobier S.A.C. ubicada en el distrito de Ventanilla, en la provincia del Callao y tuvo una duración de 41 días. Se utilizaron 16.290 pollos de carne machos de un día de edad de la línea Cobb 500, distribuidos al azar en tres grupos de 5.430 aves cada uno y estos a su vez fueron subdivididos en tres unidades experimentales con 1.810 pollos cada una. Cada grupo (5.430 aves) recibió uno de los siguientes tratamientos: T1, programa de alimentación tradicional de la empresa Gramobier S.A.C.; T2, programa de alimentación bajo sólo en calcio y fósforo disponible + suplementación con $1 \alpha-\mathrm{OHD}_{3}$ a razón de $125 \mathrm{~g}$ por tonelada métrica de alimento y $\mathbf{T} 3$, similar a $\mathrm{T} 2$, pero sin suplementación de $1 \alpha-\mathrm{OHD}_{3}$. Con excepción de calcio y fósforo disponible, las dietas experimentales fueron isocalóricas y el nivel de aminoácidos totales fue el mismo para cada una de ellas. La presentación del alimento fue en pellet y su suministro fue ad libitum. La formulación se realizó con el programa de formulación de raciones BRILL y se empleó un programa de alimentación de cuatro fases: Pre-inicio (1 a 7 días), Inicio (8 a 21 días), Crecimiento (22 a 37 días) y Acabado (38 a 41 días). La composición y el valor nutricional calculado para cada una de las dietas se presentan en las Tablas 1 y 2 . Las mediciones realizadas fueron el peso vivo, consumo voluntario de alimento, conversión alimentaria, mortalidad e incidencia de problema de patas. El estudio se realizó bajo el diseño completamente randomizado con tres tratamientos y tres repeticiones por tratamiento. Los datos obtenidos fueron sometidos a análisis de varianza usando el programa estadístico Statistical Analysis System (SAS, 1999). Para la comparación de medias se utilizó la Prueba de Duncan (Duncan, 1955). Para la estabilización de la variancia, los valores expresados en porcentajes (mortalidad y problemas de patas) fueron transformados en valores angulares, empleando la siguiente fórmula: Arcoseno $\sqrt{ }(\mathrm{Yi} / 100)$ (Steel y Torrie, 1985).

\section{Resultados y discusión}

El peso vivo final y la ganancia de peso total de cada tratamiento se presentan en la Tabla 3. Se puede observar que no hubo diferencia estadística $(\mathrm{P}>0,05)$ entre el grupo que recibió la dieta control positivo (T1) y dieta conteniendo $1 \alpha-\mathrm{OHD}_{3}$ (T2) y ambos fueron superiores $(\mathrm{P}<0,05)$ al grupo que fue alimentado con la dieta control negativo (T3). El menor peso vivo y la menor ganancia de peso observado en el grupo alimentado con la dieta T3 era de esperarse dado que la dieta era deficiente tanto en calcio como en fósforo. Por otro lado, la no diferencia entre los tratamientos T1 y T2 puede deberse a que la suplementación de la $1 \alpha-\mathrm{OHD}_{3}$ en la dieta T2 cubrió los requerimientos de calcio y fósforo similar a lo aportado por la dieta del $\mathrm{T} 1$, permitiendo el crecimiento normal de los pollos. Este resultado coincide con otros estudios realizados anteriormente en pollos de carne en los cuales se reportan incrementos en los pesos corporales cuando la $1 \alpha-\mathrm{OHD}_{3}$ es suplementada en dietas a base de maíz-soya y deficientes en calcio y fósforo (Biehl y Baker, 1997; Snow et al., 2004).

El consumo de alimento y la conversión alimenticia no fueron significativamente influenciados $(\mathrm{P}>0.05)$ por los tratamientos dietarios (Tabla 3). Sin embargo, los animales que recibieron la dieta $\mathrm{T} 3$ mostraron el consumo de alimento más bajo y esto podría deberse al menor nivel de fósforo disponible en la dieta. Al respecto, es aceptado que una deficiencia de fósforo disponible en la dieta resulta en una pérdida de apetito en las aves (Leeson y Summers, 2001). En general, en el presente estudio la suplementación de la $1 \alpha-\mathrm{OHD}_{3}$ en la dieta $\mathrm{T} 2$ no resultó en un incremento en el consumo de alimento, a diferencia de otros estudios en que sí se observaron incrementos significativos en el consumo de alimento cuando la dieta fue suplementada con $1 \alpha-\mathrm{OHD}_{3}$ (Biehl et al., 1998; Snow et al., 2004; Han et al., 2009).

En el presente estudio la suplementación de $1 \alpha-\mathrm{OHD}_{3}$ en 
Tabla 2. Composición porcentual de las dietas experimentales de crecimiento (22-37 días) y acabado (38-41 días) (justificado a la izquierda)

\begin{tabular}{|c|c|c|c|c|c|c|}
\hline \multirow[b]{2}{*}{ Ingredientes } & \multicolumn{3}{|c|}{ Crecimiento (22 - 37 días) } & \multicolumn{3}{|c|}{ Acabado (38 - 41 días) } \\
\hline & $\mathbf{T 1}^{1}$ & $\mathbf{T} 2$ & T3 & T1 & $\mathbf{T 2}$ & $\mathbf{T 3}$ \\
\hline Maíz amarillo & 67,67 & 68,25 & 68,25 & 70,97 & 71,56 & 71,56 \\
\hline Torta soya (paraguaya) & 23,92 & 24,58 & 24,58 & 21,15 & 21,81 & 21,81 \\
\hline Harina de soya integral & 4,526 & 3,543 & 3,556 & 4,396 & 3,413 & 3,426 \\
\hline Aceite de maíz & 0,5 & 0,5 & 0,5 & 0,5 & 0,5 & 0,5 \\
\hline Fosfato tricálcico & 1,09 & 0,82 & 0,82 & 0,75 & 0,47 & 0,47 \\
\hline Carbonato de calcio & 1,02 & 0,99 & 0,99 & 1,18 & 1,15 & 1,15 \\
\hline Sal común & 0,21 & 0,24 & 0,24 & 0,24 & 0,27 & 0,27 \\
\hline DL- Metionina & 0,26 & 0,26 & 0,26 & 0,2 & 0,2 & 0,2 \\
\hline Lisina HCL & 0,23 & 0,23 & 0,23 & 0,15 & 0,15 & 0,15 \\
\hline L- Treonina & 0,11 & 0,11 & 0,11 & 0,06 & 0,06 & 0,06 \\
\hline Caolín & 0,15 & 0,15 & 0,15 & 0,25 & 0,25 & 0,25 \\
\hline Lutavit 259 & 0,10 & 0,10 & 0,10 & 0,06 & 0,06 & 0,06 \\
\hline Cloruro de colina 75 & 0,07 & 0,07 & 0,07 & 0,02 & 0,02 & 0,02 \\
\hline Enzima liquido (Vegpro) & 0,05 & 005 & 0,05 & 0,05 & 0,05 & 0,05 \\
\hline Allzyme SSF & 0,02 & 0,02 & 0,02 & 0,02 & 0,02 & 0,02 \\
\hline Elancoban & 0,06 & 0,06 & 0,06 & - & - & - \\
\hline Roxarzone & 0,01 & 0,01 & 0,01 & - & - & - \\
\hline Actinospectin $11 \%$ & 0,004 & 0,004 & 0,004 & 0,004 & 0,004 & 0,004 \\
\hline Vitamina 1alpha-OHD & - & 0,0125 & - & - & 0,0125 & - \\
\hline \multicolumn{7}{|l|}{ Nutrientes (Calculado) } \\
\hline E. Metabolizable (kcal/kg) & 3.110 & 3.110 & 3.110 & 3.140 & 3.140 & 3.140 \\
\hline Proteína total, \% & 18,92 & 18,92 & 18,92 & 17,61 & 17,61 & 17,61 \\
\hline Lisina total, $\%$ & 1,18 & 1,18 & 1,18 & 1,04 & 1,04 & 1,04 \\
\hline Metionina total, $\%$ & 0,56 & 0,56 & 0,56 & 0,48 & 0,48 & 0,48 \\
\hline Metionina + Cistina total, $\%$ & 0,88 & 0,88 & 0,88 & 0,78 & 0,78 & 0,78 \\
\hline Triptófano total, \% & 0,23 & 0,23 & 0,23 & 0,22 & 0,22 & 0,22 \\
\hline Treonina total, $\%$ & 0,82 & 0,82 & 0,82 & 0,72 & 0,72 & 0,72 \\
\hline Calcio, $\%$ & 0,91 & 0,81 & 0,81 & 0,85 & 0,75 & 0,75 \\
\hline Fósforo disponible, $\%$ & 0,43 & 0,38 & 0,38 & 0,36 & 0,31 & 0,31 \\
\hline Sodio, $\%$ & 0,16 & 0,16 & 0,16 & 0,15 & 0,15 & 0,15 \\
\hline
\end{tabular}

1 Tratamientos: T1: control positivo; T2: dieta conteniendo $1 \alpha-\mathrm{OHD}_{3}$; T3: control negativo. 
Tabla 3. Comportamiento productivo en pollos de carne ( 1 a 41 días de edad)

\begin{tabular}{|c|c|c|c|}
\hline \multirow{2}{*}{ Mediciones $^{1}$} & \multicolumn{3}{|c|}{ Trat a m i ent $o^{2}$} \\
\hline & T1 & $\mathbf{T 2}$ & T3 \\
\hline Peso vivo inicial, $\mathbf{g}$ & $49^{\text {a }}$ & $48^{\text {a }}$ & $50^{a}$ \\
\hline Peso vivo final, $g$ & $2723^{a}$ & $2698^{a}$ & $2634^{b}$ \\
\hline Ganancia de peso, g & $2673^{\text {a }}$ & $2650^{a}$ & $2585^{\mathrm{b}}$ \\
\hline Consumo de alimento, $\mathrm{g}$ & $4637^{\mathrm{a}}$ & $4539^{a}$ & $4515^{\mathrm{a}}$ \\
\hline Conversión alimenticia, g/g & $1,735^{\text {a }}$ & $1,713^{\text {a }}$ & $1,747^{\mathrm{a}}$ \\
\hline Mortalidad, \% & $3,70^{\text {a }}$ & $4,20^{\text {a }}$ & $4,25^{\text {a }}$ \\
\hline
\end{tabular}

la dieta (T2) no tuvo efecto significativo $(\mathrm{P}>0,05)$ sobre la conversión alimenticia. Este resultado concuerda con los datos reportados por Edwards, Jr. et al. (2002) y Snow et al. (2004) quienes no encontraron mejoras significativas en conversión alimenticia en pollos de carne alimentados con dietas testigo y dietas suplementadas con $1 \alpha-\mathrm{OHD}_{3}$. Otras investigaciones observaron, sin embargo, efectos significativos sobre la conversión alimenticia con la suplementación de la $1 \alpha-\mathrm{OHD}_{3}$ en dietas de pollos (Han et al., 2009; Biehl y Baker, 1997). En el experimento se obtuvo, numéricamente, una mejor conversión alimenticia en el grupo que recibió la dieta T2, coincidiendo con una buena ganancia de peso de los pollos, resultados que corroboraría que la $1 \alpha-\mathrm{OHD}_{3}$ incrementa la absorción de calcio y fósforo (Leeson y Summers, 2001) permitiendo un crecimiento normal de las aves.

En este estudio la medición de la incidencia de problemas de patas en los pollos consistió en registrar en cada unidad experimental los pollos que presentaron físicamente algún tipo de deformidad en las patas o piernas. El número de pollos con problemas de patas observados en cada grupo experimental se presenta en la Tabla 4. El análisis de varianza mostró en que no existe diferencia estadística $(\mathrm{P}>0,05)$ en la incidencia de problemas de patas entre los tratamientos y también mostró un alto coeficiente de variación de 20,36 entre las unidades experimentales. Se podría afirmar en el presente estudio que la $1 \alpha-\mathrm{OHD}_{3}$ no tuvo efectos sobre la incidencia de problemas de patas en los pollos. Sin embargo, en otras investigaciones se observó incremento en la resistencia de rotura de los huesos (tibiotarso) cuando la $1 \alpha-\mathrm{OHD}_{3}$ es adicionada en dietas de pollos con bajos niveles de calcio y/o fósforo (Biehl et al., 1998; Han et al., 2009).

El alto coeficiente de variación obtenido en este experimento probablemente se deba a que la presentación de problemas de patas en los pollos no es específicamente por deficiencia de calcio y/o fósforo, sino que también hay varios factores que influyen en la presentación de este problema. Esto significa que en este estudio, los problemas de patas que presentaron los pollos de los tres tratamientos pudieron haber sido causados por factores tales como nutrición y manejo de las reproductoras, condiciones ambientales durante la incubación, calidad o procesamiento del alimento balanceado (Oviedo, 2009). Por tanto, la incidencia de problemas de patas en los pollos o número de pollos con deformidades en las patas o piernas, no es una respuesta suficientemente sensible a las dietas con deficiencias de calcio y fósforo. Asimismo, en la Tabla 3 también se aprecia que los tratamientos dietarios no influenciaron significativamente $(\mathrm{P}>0,05)$ la mortalidad de los animales.

En conclusión, bajo las condiciones en las que se ha llevado a cabo el presente trabajo de investigación, el comportamiento productivo de los pollos de carne alimentados con dietas conteniendo niveles inferiores de calcio y fósforo a los requeridos, pero suplementados con vitamina $1 \alpha-\mathrm{OHD}_{3}$, fue similar al grupo control positivo.

\section{Literatura citada}

Biehl, R. R. y Baker, D. H. 1997. Utilization of phytate and nonphytate phosphorus in chicks as affected by source and amount of vitamin $\mathrm{D}_{3}$. Journal Animal Science, 75:

Tabla 4. Cantidad de pollos con problemas de patas

\begin{tabular}{|c|c|c|c|c|}
\hline & \multicolumn{3}{|c|}{ TRATAMIENTOS ${ }^{2}$} & \multirow{2}{*}{$\begin{array}{c}\text { Coeficiente de } \\
\text { variación total, \% }\end{array}$} \\
\hline & T1 & $\mathbf{T} 2$ & T3 & \\
\hline Pollos con problemas de $\operatorname{patas}^{1}(\%)$ & $0,37^{\mathrm{a}}$ & $0,48^{\mathrm{a}}$ & $0,85^{\mathrm{a}}$ & 20,36 \\
\hline
\end{tabular}

${ }^{a, b}$ Letras distintas en una misma fila son valores diferentes estadísticamente $(P<0,05)$.

${ }^{1}$ Los valores para cada tratamiento representan el promedio de tres repeticiones.

${ }^{2}$ Tratamientos: T1: control positivo; T2: dieta conteniendo $1 \alpha-\mathrm{OHD}_{3}$; T3: control negativo. 
2986-2993.

Biehl, R.R.; Baker, D.H. y De Luca, H.F. 1998. Activity of various hydroxylated vitamin $\mathrm{D}_{3}$ analogs for improving phosphorus utilization in chicks receiving diets adequate in vitamin $\mathrm{D}_{3}$. Poultry Science, 39: 408-412.

Duncan, D.B. 1955. Multiple range and multiple F tests. Biometrics, 11, 1-42.

Edwards, H.M. Jr. 2002. Studies on the efficacy of cholecalciferol and derivatives for stimulating phytate utilization in broilers. Poultry Science, 81: 1026-1031.

Edwards, H.M. Jr.; Shirley, R.B.; Escoe, W.B. y Pesti, G.M. 2002. Quantitative evaluation of 1- $\alpha$-hydroxycholecalciferol as a cholecalciferol substitute for broilers. Poultry Science, 81: 664-669.

Han, J. C.; Yang, X.D.; Zhang, T.; Li, H.; Li, W.L.; Zhang, Y. y Yao, J.H. 2009. Effects of $1 \alpha$-hydroxycholecalciferol on growth performance, parameters of tibia and plasma, meat quality, and type IIb sodium phosphate cotransporter gene expression of one- to twenty- one-day-old broilers. Poultry Science, 88: 323-329.

Haussler, M.R.; Zerwekh, J.E.; Hesse, R.H.; Rizzardo, E. y Pechet, M.M. 1973. Biological activity of $1 \alpha$ hydroxycholecalciferol, a synthetic analog of the hormonal form of vitamin D3. Proc. Nat. Acad. Sci, 70: 2248-2252.

Liem, A.; Pesti, G.M.; Atencio. A. y Edwards, H.M. Jr. 2009. Experimental approach to optimize phytate phosphorus utilization by broiler chickens by addition of supplements. Poultry Science, 88: 1655-1665.

Oviedo, R.E. 2009. Aspectos nutricionales que influyen sobre la incidencia de problemas de patas en pollos de engorde. FEDNA. XXV Curso de Especialización (en línea). Recuperado de http://www.etsia.upm.es/fedna.com SAS Institute. 1999. SAS user's guide: Statistics, Versión 8. SAS Institute, Inc. Cary, NC.

Shim, M.; Pesti, G.M.; Bakalli, R.I. y Edwards, H.M. Jr. 2008. The effect of breeder age and egg storage time on phosphorus utilization by broiler progeny fed a phosphorus deficiency diet with $1 \alpha-\mathrm{OH}$ vitamin $\mathrm{D}_{3}$. Poultry Science, 87: 1138-1145.

Snow, J.L.; Baker, D.H. y Parsons, C.M. 2004. Phytase, citric acid, and $1 \alpha$-hydroxycholecalciferol improve phytate phosphorus utilization in chicks fed a corn-soybean meal diet. Poultry Science, 83: 1187-1192.

Steel, R.G.D. y Torrie, J H. 1985. Bioestadística: Principios y Procedimientos. (Segunda edición). México: Editorial McGraw-Hill. 